\title{
CATEGORIFICATION OF SIGN-SKEW-SYMMETRIC CLUSTER ALGEBRAS AND SOME CONJECTURES ON g-VECTORS
}

\author{
PEIGEN CAO MIN HUANG FANG LI
}

\begin{abstract}
Using the unfolding method given in [12, we prove the conjectures on sign-coherence and a recurrence formula respectively of $\mathbf{g}$-vectors for acyclic sign-skew-symmetric cluster algebras. As a following consequence, the conjecture is affirmed in the same case which states that the $\mathrm{g}$ vectors of any cluster form a basis of $\mathbb{Z}^{n}$. Also, the additive categorification of an acyclic sign-skewsymmetric cluster algebra $\mathcal{A}(\Sigma)$ is given, which is realized as $\left(\mathcal{C}^{\widetilde{Q}}, \Gamma\right)$ for a Frobenius 2-Calabi-Yau category $\mathcal{C}^{\widetilde{Q}}$ constructed from an unfolding $(Q, \Gamma)$ of the acyclic exchange matrix $B$ of $\mathcal{A}(\Sigma)$.
\end{abstract}

\section{INTRODUCTION}

Cluster category was introduced by [2] for an acyclic quiver. In general, we view a Hom-finite 2-Calabi-Yau triangulated category $\mathcal{C}$ which has a cluster structure as a cluster category, see [1. In fact, the mutation of a cluster-tilting object $T$ in $\mathcal{C}$ categorifies the mutation of a quiver $Q$, where the quiver $Q$ is the Gabriel quiver of the algebra $\operatorname{End}_{\mathcal{C}}(T)$. Cluster character gives an explicit correspondence between certain cluster objects of $\mathcal{C}$ and all the clusters of $\mathcal{A}(\Sigma(Q))$, where $\Sigma(Q)$ means the seed associated with $Q$. For details, see [10, [14] and [15]. Thus, cluster category and cluster character are useful tools to study a cluster algebra.

Let $\mathcal{A}(\Sigma)$ be a cluster algebra with principal coefficients at $\Sigma=(X, Y, B)$, where $B$ is an $n \times n$ sign-skew-symmetric integer matrix, $Y=\left(y_{1}, \cdots, y_{n}\right)$. The celebrate Laurent phenomenon says that $\mathcal{A}$ is a subalgebra of $\mathbb{Z}\left[y_{n+1}, \cdots, y_{2 n}\right]\left[X^{ \pm 1}\right]$. Setting $\operatorname{deg}\left(x_{i}\right)=e_{i}, \operatorname{deg}\left(y_{j}\right)=-b_{j}$, then $\mathbb{Z}\left[y_{n+1}, \cdots, y_{2 n}\right]\left[X^{ \pm 1}\right]$ becomes to a graded algebra, where $\left\{e_{i} \mid i=1, \cdots, n\right\}$ is the standard basis of $\mathbb{Z}^{n}$ and $b_{i}$ is the $i$-th column of $B$. Under such $\mathbb{Z}^{n}$-grading, the cluster algebra $\mathcal{A}$ is a graded subalgebra, in which the degree of a homogenous element is called its g-vector; furthermore, each cluster variable $x$ in $\mathcal{A}(\Sigma)$ is homogenous, denoted its $\mathbf{g}$-vector as $\mathbf{g}(x)$. For details, see 9 .

It was conjectured that

Conjecture 1.1. (9], Conjecture 6.13) For any cluster $X^{\prime}$ of $\mathcal{A}(\Sigma)$ and all $x \in X^{\prime}$, the vectors $\mathbf{g}(x)$ are sign-coherent, which means that the $i$-th coordinates of all these vectors are either all non-negative or all non-positive.

Such conjecture has been proved in the skew-symmetrizable case in ([11, Theorem 5.11).

Conjecture 1.2. (9], Conjecture 7.10(2)) For any cluster $X^{\prime}$ of $\mathcal{A}(\Sigma)$, the vectors $\mathrm{g}(x), x \in X^{\prime}$ form a $\mathbb{Z}$-basis of the lattice $\mathbb{Z}^{n}$.

In terms of cluster pattern, fixed a regular tree $\mathbb{T}_{n}$, for any vertices $t$ and $t_{0}$ of $\mathbb{T}_{n}$, let $\mathbf{g}_{1 ; t}^{B^{0} ; t_{0}}, \cdots, \mathbf{g}_{n ; t}^{B^{0} ; t_{0}}$ denote the $\mathbf{g}$-vectors of the cluster variables in the seed $\Sigma_{t}$ with respect to the principal coefficients seed $\Sigma_{t_{0}}=\left(X_{0}, Y, B_{0}\right)$. When set different vertices to be principal coefficients seeds, it is conjectured that the g-vectors with respect to a fixed vertex $t$ of $\mathbb{T}_{n}$ have the following relation.

Date: version of November 10, 2021. 


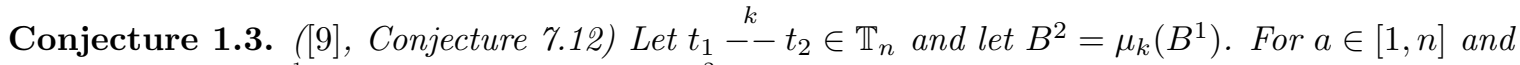
$t \in \mathbb{T}_{n}$, assume $\mathbf{g}_{a ; t}^{B^{1} ; t_{1}}=\left(g_{1}^{t_{1}}, \cdots, g_{n}^{t_{1}}\right)$ and $\mathbf{g}_{a ; t}^{B^{2} ; t_{2}}=\left(g_{1}^{t_{2}}, \cdots, g_{n}^{t_{2}}\right)$, then

$$
g_{i}^{t_{2}}= \begin{cases}-g_{k}^{t_{1}} & \text { if } i=k \\ g_{i}^{t_{1}}+\left[b_{i k}^{t_{1}}\right]_{+} g_{k}^{t_{1}}-b_{i k}^{t_{1}} \min \left(g_{k}^{t_{1}}, 0\right) & \text { if } i \neq k\end{cases}
$$

Remark 1.4. (1) As is said in Remark 7.14 of [9], it is easy to see that Conjectures 1.1 and 1.3 imply Conjecture 1.2

(2) In the skew-symmetrizable case, the sign-coherence of the c-vectors can deduce Conjectures 1.1 and 1.3 see [13, where the given method strongly depends on the skew-symmetrizability. So far, the similar conclusion have not been given in the sign-skew-symmetric case. Further, the signcoherence of the c-vectors has been proved in [12] for the acyclic sign-skew-symmetric case, because this is equivalent to $F$-polynomial has constant term 1, see [9]. Therefore, for the acyclic sign-skewsymmetric case, it is interesting to study directly Conjectures 1.1 and 1.3 .

Unfolding of skew-symmetrizable matrices is introduced by Zelevinsky, whose aim is to characterize skew-symmetrizable cluster algebras using the version in skew-symmetric case. The second and third authors of this paper improved in [12] such method to arbitrary sign-skew-symmetric matrices. According to this previous work, there is an unfolding $(\widetilde{Q}, F, \Gamma)$ of any acyclic $m \times n$ matrix $\widetilde{B}$, and also a 2-Calabi-Yau Frobenius category $\mathcal{C}^{\widetilde{Q}}$ with $\Gamma$ action constructed.

Our motivation and the main results of this paper are two-fold.

(1) Give the $\Gamma$-equivariant cluster character for $\underline{\mathcal{C}^{Q}}$, which can be regarded as the additive categorification of the cluster algebra $\mathcal{A}(\Sigma(Q))$. See Theorem 3.5 and Theorem 3.6.

(2) Solve Conjectures 1.1 and 1.3 in the acyclic case. See Theorem 4.7 and Theorem [5.5. As a consequence, in the same case, Conjecture 1.2 follows to be affirmed.

\section{AN OVERVIEW OF UNFOLDING METHOD}

In this section, we give a brief introduction of the concept of unfolding of totally sign-skewsymmetric cluster algebras and some necessary results in [12.

For any sign-skew-symmetric matrix $B \in \operatorname{Mat}_{n \times n}(\mathbb{Z})$, one defines a quiver $\Delta(B)$ as follows: the vertices are $1, \cdots, n$ and there is an arrow from $i$ to $j$ if and only if $b_{i j}>0$. B is called acyclic if $\Delta(B)$ is acyclic, a cluster algebra is called acyclic if it has an acyclic exchange matrix, see [3].

A locally finite ice quiver is a pair $(Q, F)$ where $Q$ is a locally finite quiver without 2-cycles or loops and $F \subseteq Q_{0}$ is a subset of vertices called frozen vertices such that there are no arrows among vertices of $F$. For a locally finite ice quiver $(Q, F)$, we can associate an (infinite) skewsymmetric row and column finite (i.e. having at most finite nonzero entries in each row and column) matrix $\left(b_{i j}\right)_{i \in Q_{0}, j \in Q_{0} \backslash F}$, where $b_{i j}$ equals to the number of arrows from $i$ to $j$ minus the number of arrows from $j$ to $i$. In case of no confusion, for convenience, we also denote the ice quiver $(Q, F)$ as $\left(b_{i j}\right)_{i \in Q_{0}, j \in Q_{0} \backslash F}$.

We say that an ice quiver $(Q, F)$ admits the action of a group $\Gamma$ if $\Gamma$ acts on $Q$ such that $F$ is stable under the action. Let $(Q, F)$ be a locally finite ice quiver with an action of a group $\Gamma$ (maybe infinite). For a vertex $i \in Q_{0} \backslash F$, a $\Gamma$-loop at $i$ is an arrow from $i$ to $h \cdot i$ for some $h \in \Gamma$, a $\Gamma$-2-cycle at $i$ is a pair of arrows $i \rightarrow j$ and $j \rightarrow h \cdot i$ for some $j \notin\left\{h^{\prime} \cdot i \mid h^{\prime} \in \Gamma\right\}$ and $h \in \Gamma$. Denote by $[i]$ the orbit set of $i$ under the action of $\Gamma$. Say that $(Q, F)$ has no $\Gamma$-loops (respectively, $\Gamma$-2-cycles) at $[i]$ if $(Q, F)$ has no $\Gamma$-loops $\left(\Gamma\right.$-2-cycles, respectively) at any $i^{\prime} \in[i]$. 
Definition 2.1. (Definition 2.1, [12]) Let $(Q, F)=\left(b_{i j}\right)$ be a locally finite ice quiver with an group $\Gamma$ action. Denote $[i]=\{h \cdot i \mid h \in \Gamma\}$ the orbit of vertex $i \in Q_{0} \backslash F$. Assume that $(Q, F)$ admits no $\Gamma$-loops and no $\Gamma$-2-cycles at $[i]$, we define an adjacent ice quiver $\left(Q^{\prime}, F\right)=\left(b_{i^{\prime} j^{\prime}}^{\prime}\right)_{i^{\prime} \in Q_{0}, j^{\prime} \in Q_{0} \backslash F}$ from $(Q, F)$ to be the quiver by following:

(1) The vertices are the same as $Q$,

(2) The arrows are defined as

$$
b_{j k}^{\prime}= \begin{cases}-b_{j k}, & \text { if } j \in[i] \text { or } k \in[i], \\ b_{j k}+\sum_{i^{\prime} \in[i]} \frac{\left|b_{j i^{\prime}}\right| b_{i^{\prime} k}+b_{j i^{\prime}}\left|b_{i^{\prime} k}\right|}{2}, & \text { otherwise. }\end{cases}
$$

Denote $\left(Q^{\prime}, F\right)$ as $\widetilde{\mu}_{[i]}((Q, F))$ and call $\widetilde{\mu}_{[i]}$ the orbit mutation at direction $[i]$ or at $i$ under the action $\Gamma$. In this case, we say that $(Q, F)$ can do orbit mutation at $[i]$.

Note that if $\Gamma$ is the trivial group $\{e\}$, then the definition of orbit mutation of a quiver is the same as that of quiver mutation (see [7] [8]).

Definition 2.2. (Definition 2.4, [12]) (i) For a locally finite ice quiver $(Q, F)=\left(b_{i j}\right)_{i \in Q_{0}, j \in Q_{0} \backslash F}$ with a group $\Gamma$ (maybe infinite) action, let $\overline{Q_{0}}$ (respectively, $\bar{F}$ ) be the orbit sets of the vertex set $Q_{0}$ (respectively, the frozen vertex set $F$ ) under the $\Gamma$-action. Assume that $m=\left|\overline{Q_{0}}\right|<+\infty, m-n=|\bar{F}|$ and $Q$ has no $\Gamma$-loops and no $\Gamma$-2-cycles.

Define a sign-skew-symmetric matrix $B(Q, F)=\left(b_{[i][j]}\right)_{[i] \in \overline{Q_{0}},[j] \in \overline{Q_{0}} \backslash \bar{F}}$ to $(Q, F)$ satisfying $(1)$ the size of the matrix $B(Q, F)$ is $m \times n ;(2) b_{[i][j]}=\sum_{i^{\prime} \in[i]} b_{i^{\prime} j}$ for $[i] \in \overline{Q_{0}},[j] \in \overline{Q_{0}} \backslash \bar{F}$.

(ii) For an $m \times n$ sign-skew-symmetric matrix $B$, if there is a locally finite ice quiver $(Q, F)$ with a group $\Gamma$ such that $B=B(Q, F)$ as constructed in (i), then we call $(Q, F, \Gamma)$ a covering of $B$.

(iii) For an $m \times n$ sign-skew-symmetric matrix $B$, if there is a locally finite quiver $(Q, F)$ with an action of group $\Gamma$ such that $(Q, F, \Gamma)$ is a covering of $B$ and $(Q, F)$ can do arbitrary steps of orbit mutations, then $(Q, F, \Gamma)$ is called an unfolding of $B$; or equivalently, $B$ is called the folding of $(Q, F, \Gamma)$.

Remark 2.3. The definition of unfolding is slight different with that in [12] where the definition was just applied to square matrices.

By Lemma 2.5 of [12, we have the following consequence.

Lemma 2.4. If $(Q, F, \Gamma)$ is an unfolding of $B$, for any sequence $\left[i_{1}\right], \cdots,\left[i_{s}\right]$ of orbits of $Q_{0} \backslash F$ under the action of $\Gamma$, then $\left(\widetilde{\mu}_{\left[i_{s}\right]} \cdots \widetilde{\mu}_{\left[i_{s}\right]}(Q, F), \Gamma\right)$ is a covering of $\mu_{\left[i_{s}\right]} \cdots \mu_{\left[i_{1}\right]} B$.

By Theorem 2.16 of [12, we have

Theorem 2.5. If $\widetilde{B} \in M_{a} t_{m \times n}(\mathbb{Z})(m \geq n)$ is an acyclic sign-skew-symmetric matrix, then $\widetilde{B}$ has an unfolding $(\widetilde{Q}, F, \Gamma)$, where $\widetilde{Q}$ is given using of Construction 2.6 in [12].

Proof. Assume $\widetilde{B}=\left(\begin{array}{c}B \\ B^{\prime}\end{array}\right)$ with $B \in \operatorname{Mat}_{n \times n}(\mathbb{Z})$. Denote $\widetilde{B}^{\prime}=\left(\begin{array}{cc}B & -B^{\prime T} \\ B^{\prime} & 0\end{array}\right)$. Since $\widetilde{B}$ is acyclic, $\widetilde{B}^{\prime} \in \operatorname{Mat}_{m \times m}(\mathbb{Z})$ is acyclic. According to Construction 2.6 and Theorem 2.16 of [12], $\widetilde{B}^{\prime}$ has an unfolding $(\widetilde{Q}, \Gamma)$. Let $F \subset \widetilde{Q}_{0}$ be the vertices of $\widetilde{Q}_{0}$ corresponding to $B^{\prime}$. Thus, it is clear that $(\widetilde{Q}, F, \Gamma)$ is an unfolding of $\widetilde{B}$.

Remark 2.6. In [12], we proved that $\widetilde{Q}$ is strongly almost finite, that is, $\widetilde{Q}$ is locally finite and has no path of infinite length. 
This theorem means that an acyclic matrix is always totally sign-skew-symmetric. Thus, we can define a cluster algebra via an acyclic matrix.

For an acyclic matrix $\widetilde{B} \in M a t_{m \times n}(\mathbb{Z})(m \geq n)$, assume $(\widetilde{Q}, F, \Gamma)$ is an unfolding of $\widetilde{B}$. Denote $\overline{\widetilde{Q}_{0}}$ and $\bar{F}$ be the orbits sets of vertices in $\widetilde{Q}_{0}$ and $F$. Let $\widetilde{\Sigma}=\Sigma(\widetilde{Q})=(\widetilde{X}, \widetilde{Y}, \widetilde{Q})$ be the seed associated with $(\widetilde{Q}, F)$, where $\widetilde{X}=\left\{x_{u} \mid u \in \widetilde{Q}_{0} \backslash F\right\}, \widetilde{Y}=\left\{y_{v} \mid v \in F\right\}$. Let $\Sigma=\Sigma(\widetilde{B})=(X, Y, \widetilde{B})$ be the seed associated with $\widetilde{B}$, where $X=\left\{x_{[i]} \mid[i] \in{\widetilde{Q_{0}}}_{\bar{F}}\right\}, Y=\left\{y_{[j]} \mid[j] \in \bar{F}\right\}$. It is clear that there is a surjective algebra homomorphism:

$$
\pi: \mathbb{Q}\left[x_{i}^{ \pm 1}, y_{j} \mid i \in \widetilde{Q}_{0} \backslash F, j \in F\right] \rightarrow \mathbb{Q}\left[x_{[i]}^{ \pm 1}, y_{[j]} \mid[i] \in \widetilde{Q}_{0} \backslash \bar{F},[j] \in \bar{F}\right]
$$

such that $\pi\left(x_{i}\right)=x_{[i]}$ and $\pi\left(y_{j}\right)=y_{[j]}$.

For any cluster variable $x_{u} \in \widetilde{X}$, define $\widetilde{\mu}_{[i]}\left(x_{u}\right)=\mu_{u}\left(x_{u}\right)$ if $u \in[i]$; otherwise, $\widetilde{\mu}_{[i]}\left(x_{u}\right)=x_{u}$ if $u \notin[i]$. Formally, write $\widetilde{\mu}_{[i]}(\widetilde{X})=\left\{\widetilde{\mu}_{[i]}(x) \mid x \in \widetilde{X}\right\}$ and $\widetilde{\mu}_{[i]}\left(\widetilde{X}^{ \pm 1}\right)=\left\{\widetilde{\mu}_{[i]}(x)^{ \pm 1} \mid x \in \widetilde{X}\right\}$.

Lemma 2.7. (Lemma 7.1, [12]) Keep the notations as above. Assume that B is acyclic. If $[i]$ is an orbit of vertices with $i \in \widetilde{Q}_{0} \backslash F$, then

(1) $\widetilde{\mu}_{[i]}\left(x_{j}\right)$ is a cluster variable of $\mathcal{A}(\widetilde{Q})$ for any $j \in \widetilde{Q}_{0} \backslash F$,

(2) $\widetilde{\mu}_{[i]}(\widetilde{X})$ is algebraic independent over $\mathbb{Q}\left[y_{j} \mid j \in F\right]$.

By Lemma 2.7 $\widetilde{\mu}_{[i]}(\widetilde{\Sigma}):=\left(\widetilde{\mu}_{[i]}(\widetilde{X}), \widetilde{Y}, \widetilde{\mu}_{[i]}(\widetilde{Q})\right)$ is a seed. Thus, we can define $\widetilde{\mu}_{\left[i_{s}\right]} \widetilde{\mu}_{\left[i_{s-1}\right]} \cdots \widetilde{\mu}_{\left[i_{1}\right]}(x)$ and $\widetilde{\mu}_{\left[i_{s}\right]} \widetilde{\mu}_{\left[i_{s-1}\right]} \cdots \widetilde{\mu}_{\left[i_{1}\right]}(\widetilde{X})$ and $\widetilde{\mu}_{\left[i_{s}\right]} \widetilde{\mu}_{\left[i_{s-1}\right]} \cdots \widetilde{\mu}_{\left[i_{1}\right]}(\widetilde{\Sigma})$ for any sequence $\left(\left[i_{1}\right],\left[i_{2}\right], \cdots,\left[i_{s}\right]\right)$ of orbits in $Q_{0}$.

Theorem 2.8. (Theorem 7.5, [12]) Keep the notations as above with an acyclic sign-skew-symmetric matrix $B$ and $\pi$ as defined in (2). Restricting $\pi$ to $\mathcal{A}(\widetilde{\Sigma})$, then $\pi: \mathcal{A}(\widetilde{\Sigma}) \rightarrow \mathcal{A}(\Sigma)$ is a surjective algebra morphism satisfying that $\pi\left(\widetilde{\mu}_{\left[j_{k}\right]} \cdots \widetilde{\mu}_{\left[j_{1}\right]}\left(x_{a}\right)\right)=\mu_{\left[j_{k}\right]} \cdots \mu_{\left[j_{1}\right]}\left(x_{[i]}\right) \in \mathcal{A}(\Sigma)$ and $\pi\left(\widetilde{\mu}_{\left[j_{k}\right]} \cdots \widetilde{\mu}_{\left[j_{1}\right]}(\widetilde{X})\right)=$ $\mu_{\left[j_{k}\right]} \cdots \mu_{\left[j_{1}\right]}(X)$ for any sequences of orbits $\left[j_{1}\right], \cdots,\left[j_{k}\right]$ and any $a \in[i]$.

In case $\mathcal{A}(\Sigma)$ with principal coefficients, from Lemma 2.4 , we may assume that $\mathcal{A}(\widetilde{\Sigma})$ is also with principal coefficients. Let $\lambda: \underset{i \in \widetilde{Q}_{0} \backslash F}{\bigoplus} \mathbb{Z} e_{i} \rightarrow \underset{[i] \in \widetilde{Q}_{0} \backslash \bar{F}}{\bigoplus} \mathbb{Z} e_{[i]}, e_{i} \rightarrow e_{[i]}$ be the group homomorphism, where $\bigoplus_{\widetilde{Q}} \mathbb{Z} e_{i}$ (resp. $\left.\bigoplus_{\widetilde{Q}} \mathbb{Z} e_{[i]}\right)$ is the free abelian group generated by $\left\{e_{i} \mid i \in \widetilde{Q}_{0} \backslash F\right\}$ (resp. $i \in \widetilde{Q}_{0} \backslash F \quad[i] \in \widetilde{\widetilde{Q}}_{0} \backslash \bar{F}$

$\left.\left\{e_{[i]} \mid[i] \in \overline{\widetilde{Q}_{0}} \backslash \bar{F}\right\}\right)$. Under such group homomorphism, $\mathcal{A}(\widetilde{\Sigma})$ becomes a $\mathbb{Z}^{n}$-graded algebra such any cluster variable $x$ is homogenous with degree $\lambda(\mathbf{g}(x))$.

Theorem 2.9. Keep the notations as in Theorem 2.8. If $\mathcal{A}(\Sigma)$ with principal coefficients, then the restriction of $\pi$ to $\mathcal{A}(\widetilde{\Sigma})$ is a $\mathbb{Z}^{n}$-graded surjective homomorphism.

Proof. Since $\lambda\left(\mathbf{g}\left(x_{i}\right)\right)=e_{[i]}=\mathbf{g}\left(x_{[i]}\right)$ and $\lambda\left(\mathbf{g}\left(y_{j}\right)\right)=-b_{[j]}=\mathbf{g}\left(y_{[j]}\right)$, where $b_{[j]}$ is the $[j]$-th column of $B$. Further, because $\left\{x_{i}^{ \pm 1}, y_{j} \mid i \in \widetilde{Q}_{0} \backslash F, j \in \widetilde{Q}_{0}\right\}$ is a generator of $\mathbb{Q}\left[x_{i}^{ \pm 1}, y_{j} \mid i \in \widetilde{Q}_{0} \backslash F, j \in \widetilde{Q}_{0}\right]$, thus $\pi$ is homogenous. Then our result follows by Theorem 2.8 .

\section{Cluster Character in Sign-SKeW-SymmetriC CASE}

Let $\widetilde{B} \in \operatorname{Mat}_{m \times n}(\mathbb{Z})$ be an acyclic sign-skew-symmetric matrix, $(\widetilde{Q}, F, \Gamma)$ be an unfolding of $\widetilde{B}$ given in Theorem 2.5. Denote $\widetilde{\Sigma}=(\widetilde{X}, \widetilde{Y}, \widetilde{Q})$ and $\Sigma=(X, Y, \widetilde{B})$ be the seeds corresponding to $(\widetilde{Q}, F)$ and $\widetilde{B}$.

From $(\widetilde{Q}, F, \Gamma)$, we constructed a 2-Calabi-Yau Frobenius category $\mathcal{C}^{\widetilde{Q}}$ in [12] such that $\Gamma$ acts on it exactly, i.e. each $h \in \Gamma$ acts on $\mathcal{C}^{\widetilde{Q}}$ as an exact functor. Furthermore, there exists a cluster tilting subcategory $\mathcal{T}_{0}$ of $\mathcal{C}^{\widetilde{Q}}$ such that the Gabriel quiver of $\underline{\mathcal{T}_{0}}$ is isomorphic to $\widetilde{Q}$, where $\underline{\mathcal{T}_{0}}$ is the 
subcategory of the stable category $\underline{\mathcal{C}} \underline{\underline{Q}}$ corresponding to $\mathcal{T}_{0}$. For details, see Lemma 4.15 of 12 . Since $\mathcal{C}^{\widetilde{Q}}$ is a Hom-finite 2-Calabi-Yau Frobenius category, $\underline{\mathcal{C}^{\widetilde{Q}}}$ follows to be a $H o m$-finite 2-Calabi-Yau triangulated category. Write [1] as the shift functor in $\underline{\mathcal{C}^{\widetilde{Q}}}$. For any object $X$ and subcategory $\mathcal{X}$ of $\mathcal{C}^{\widetilde{Q}}$, we denote by $\underline{X}$ and $\underline{\mathcal{X}}$ the corresponding object and subcategory of $\underline{\mathcal{C}^{\widetilde{Q}}}$ respectively. Since the action of the group $\Gamma$ on $\mathcal{C}^{\widetilde{Q}}$ is exact, $\underline{\mathcal{C}} \widetilde{Q}$ also admits an exact $\Gamma$-action.

Since $\mathcal{C}^{\widetilde{Q}}$ is a Frobenius category, by the standard result, see [1], we have that

Lemma 3.1. $\left.\operatorname{Ext}_{\mathcal{C}^{\widetilde{Q}}}^{1}\left(Z_{1}, Z_{2}\right) \cong \operatorname{Ext}_{\underline{\mathcal{C} \widetilde{Q}}}^{1} \underline{\left(Z_{1}\right.}, \underline{Z_{2}}\right)$ for all $Z_{1}, Z_{2} \in \mathcal{C}^{\widetilde{Q}}$.

The category $\underline{\mathcal{C}^{\widetilde{Q}}}$ can be viewed as an additive categorification of the cluster algebra $\mathcal{A}(\widetilde{\Sigma})$ given from $\widetilde{Q}$. For details, refer to [10] and [15]. Although the authors of [10] and [15] deal with the cluster algebras of finite ranks, it is easy to see that these results still hold in $\underline{\mathcal{C}^{\widetilde{Q}}}$ since $Q^{\prime \prime}$, as well as $\widetilde{Q}$, is a strongly almost finite quiver.

Denote $\underline{\mathcal{T}_{0}}=\operatorname{add}\left(\underline{\mathcal{T}^{\prime}} \cup \underline{\mathcal{T}^{\prime \prime}}\right)$, where $\underline{\mathcal{T}^{\prime}}$ and $\underline{\mathcal{T}^{\prime \prime}}$ respectively consist of the indecomposable objects correspondent to cluster variables in the clusters $\widetilde{X}$ and $\widetilde{Y}$ of $\widetilde{\Sigma}$.

Let $\mathcal{U}$ be the subcategory of $\underline{\mathcal{C}^{\widetilde{Q}}}$ generated by $\left\{\underline{X} \in \underline{\mathcal{C}^{\widetilde{Q}}} \mid \operatorname{Hom}_{\underline{\mathcal{C}^{\tilde{Q}}}}(\underline{T}[-1], \underline{X})=0, \forall \underline{T} \in \underline{\mathcal{T}^{\prime \prime}}\right\}$.

For any $\underline{X} \in \mathcal{U}$, let $\underline{T}_{1} \rightarrow \underline{T}_{0} \stackrel{f}{\rightarrow} \underline{X} \rightarrow \underline{T}_{1}[1]$ be the triangle with $f$ the minimal right $\underline{\mathcal{T}}^{-}$ approximation. By Lemma 3.1, $\underline{\mathcal{T}}_{0}$ is a cluster tilting subcategory of $\underline{\mathcal{C}} \tilde{Q}$. Applying $\operatorname{Hom}_{\underline{\mathcal{C}} \tilde{\underline{Q}}}(\underline{T},-)$ to the triangle for all $\underline{T} \in \underline{\mathcal{T}_{0}}$, we have $\underline{T}_{1} \in \underline{\mathcal{T}_{0}}$. The index of $\underline{X}$ is defined as

$$
\operatorname{ind}_{\underline{\mathcal{T}_{0}}}(\underline{X})=\left[\underline{T}_{0}\right]-\left[\underline{T}_{1}\right] \in K_{0}\left(\underline{\mathcal{T}_{0}}\right) \cong \mathbb{Z}^{\left|\widetilde{Q}_{0}\right|} .
$$

Recall that the Gabriel quiver of $\underline{\mathcal{T}_{0}}$ is isomorphic to $\widetilde{Q}$. We may assume that $\left\{X_{i} \mid i \in\right.$ $\left.\widetilde{Q}_{0}\right\}$ is the complete set of the indecomposable objects of $\underline{\mathcal{T}_{0}}$. For any $L \in \bmod \underline{\mathcal{T}_{0}}$, we denote $\left(\operatorname{dim}_{k}\left(L\left(X_{i}\right)\right)\right)_{i \in \widetilde{Q}_{0}} \in \bigoplus_{i \in \widetilde{Q}_{0}} \mathbb{Z}^{e_{i}}$ as its dimensional vector.

After the preparations, we give the definition of cluster character $C C(\quad)$ on $\underline{\mathcal{C} \widetilde{Q}}$. For any $i \in \widetilde{Q}_{0} \backslash F$, since $\widetilde{Q}$ is strongly almost finite, we can set

$$
\widehat{y}_{i}=\prod_{j \in \widetilde{Q}_{0} \backslash F} x_{j}^{b_{j i}} \prod_{j^{\prime} \in F} y_{j^{\prime}}^{b_{j^{\prime} i}} \in \mathbb{Q}\left[x_{i}^{ \pm 1}, y_{j} \mid i \in \widetilde{Q}_{0} \backslash F, j \in F\right] .
$$

For each rigid object $\underline{X} \in \mathcal{U}$, we define

$$
C C(\underline{X})=\widetilde{\mathbf{x}}^{i n d_{\mathcal{T}_{0}}}(\underline{X}) \sum_{\mathbf{a} \in \bigoplus_{i \in \widetilde{Q}_{0}} \mathbb{Z} e_{i}} \chi\left(G r_{\mathbf{a}}\left(\operatorname{Hom}_{\underline{\mathcal{C} \tilde{Q}}}(-, \underline{X}[1])\right)\right) \prod_{j \in \widetilde{Q}_{0} \backslash F} \widehat{y}_{j}^{a_{j}},
$$

where $\widetilde{\mathbf{x}}^{\mathbf{a}}=\Pi x_{i}^{a_{i}}$ for $\mathbf{a}=\left(a_{i}\right)_{i \in \widetilde{Q}_{0}} \in \underset{i \in \widetilde{Q}_{0}}{\bigoplus} \mathbb{Z} e_{i}, \operatorname{Gr}_{\mathbf{a}}\left(\operatorname{Hom}_{\underline{\mathcal{C}}}(-, \underline{X}[1])\right)$ is the quiver Grassmannian whose points are corresponding to the sub- $\underline{\mathcal{T}}_{0}$-representations of $H_{o m} \underline{\underline{\mathcal{C}^{\tilde{Q}}}}(-, \underline{X}[1])$ with dimension vector $\mathbf{a}$, and $\chi$ is the Euler characteristic with respect to étale cohomology with proper support. It is easy to see that $C C(\underline{X}) \in \mathbb{Q}\left[x_{i}^{ \pm 1}, y_{j} \mid i \in \widetilde{Q}_{0} \backslash F, j \in F\right]$.

Theorem 3.2. Keeps the notations as above. Then

(1) $C C\left(\underline{T_{i}}\right)=x_{i}$ for all $\underline{T_{i}} \in \underline{\mathcal{T}^{\prime}}$.

(2) $C C\left(\underline{X} \oplus \underline{X}^{\prime}\right)=C C(\underline{X}) C C\left(\underline{X}^{\prime}\right)$ for any objects $\underline{X}, \underline{X}^{\prime} \in \mathcal{U}$.

(3) $C C(\underline{X}) C C(\underline{Y})=C C(\underline{Z})+C C\left(\underline{Z}^{\prime}\right)$ for $\underline{X}, \underline{Y} \in \mathcal{U}$ with $\operatorname{dim} \operatorname{Ext}_{\mathcal{C}^{\tilde{Q}}}^{1}(\underline{X}, \underline{Y})=1$ and the two non-splitting triangles: $\underline{Y} \rightarrow \underline{Z} \rightarrow \underline{X} \rightarrow \underline{Y}[1]$ and $\underline{X} \rightarrow \underline{Z^{\prime}} \rightarrow \underline{Y} \rightarrow \underline{X}[1]$.

Proof. This theorem can be proved similarly as that of ([10, Theorem 3.3) using of local finiteness of $\widetilde{Q}$ since it is strongly almost finite. 
Like [5], for any $\underline{X} \in \mathcal{U}$, we define $i n d_{\underline{\mathcal{T}_{0}}}^{\prime}(\underline{X})=\lambda\left(i n{\underline{\mathcal{T}_{0}}}_{\underline{X}}(\underline{X})\right) \in \underset{[i] \in{\widetilde{\widetilde{Q}_{0}}}^{\bigoplus}}{\bigoplus \mathbb{Z}} e_{[i]}$.

For each rigid object $\underline{X} \in \mathcal{U}$, using the definition of $\pi$ in (2), we define the $\Gamma$-equivariant cluster character as follows:

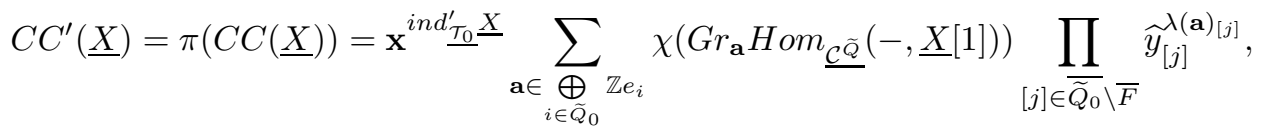

where $\mathbf{x}^{\mathbf{a}}=\Pi x_{[i]}^{\left.a_{[i]}\right]}$ for $\mathbf{a}=\left(a_{[i]}\right)_{[i] \in \widetilde{Q}_{0} \backslash \bar{F}} \in \underset{[i] \in \widetilde{\widetilde{Q}_{0} \backslash \bar{F}}}{\bigoplus} \mathbb{Z} e_{[i]}$ and $\widehat{y}_{[i]}=\pi\left(\widehat{y}_{i}\right)=\prod_{[j] \in \widetilde{Q}_{0} \backslash \bar{F}} x_{[j]}^{b_{[j]] i]}} \prod_{\left[j^{\prime}\right] \in \widetilde{Q}_{0} \backslash \bar{F}} y_{\left[j^{\prime}\right]}^{b_{\left[j^{\prime}\right][i]}}$.

Inspired by Definition 3.34 of [5], we give the following definition,

Definition 3.3. Two objects $\underline{X}, \underline{X}^{\prime} \in \underline{\mathcal{C}^{\widetilde{Q}}}$ are said to be equivalent modulo $\Gamma$ if $\underline{X} \cong \bigoplus_{k=1}^{m} \underline{X}_{k}, \underline{X}^{\prime} \cong$ $\bigoplus_{k=1}^{m} \underline{X}_{k}^{\prime}$ with $a d d\left\{h \cdot \underline{X}_{k} \mid h \in \Gamma\right\}=\operatorname{add}\left\{h \cdot \underline{X}_{k}^{\prime} \mid h \in \Gamma\right\}$ for every $k$ and indecomposables $\underline{X}_{k}, \underline{X}_{k}^{\prime}$.

Similar to Lemma 3.49 of [5], we have the following lemma,

Lemma 3.4. The Laurent polynomial $C C^{\prime}(\underline{X})$ depends only on the class of $\underline{X}$ under equivalent modulo $\Gamma$ for and $\underline{X} \in \underline{\mathcal{C}^{\widetilde{Q}}}$.

Proof. We need only to prove that $C C^{\prime}(\underline{X})=C C^{\prime}(h \cdot \underline{X})$ for $h \in \Gamma$ by Theorem $3.2(2)$. It follows

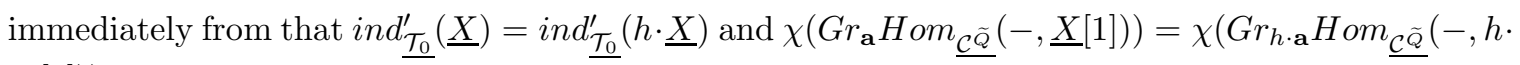
$\underline{X}[1]))$.

Using the algebra homomorphism $\pi$ and Theorem 3.2, we have the following theorem at once,

Theorem 3.5. Keeps the notations as above. Then

(1) $C C^{\prime}\left(\underline{T_{i}}\right)=x_{[i]}$ for all $\underline{T}_{i} \in \underline{\mathcal{T}}^{\prime}$.

(2) $C C^{\prime}\left(\underline{X} \oplus \underline{X}^{\prime}\right)=C C^{\prime}(\underline{X}) C C^{\prime}\left(\underline{X}^{\prime}\right)$ for any two objects $\underline{X}, \underline{X^{\prime}} \in \mathcal{U}$.

(3) $C C^{\prime}(\underline{X}) C C^{\prime}(\underline{Y})=C C^{\prime}(\underline{Z})+C C^{\prime}\left(\underline{Z}^{\prime}\right)$ for $\underline{X}, \underline{Y} \in \mathcal{U}$ with $\operatorname{dimExt} t_{\mathcal{C} \widetilde{Q}}^{1}(\underline{X}, \underline{Y})=1$ satisfying two non-splitting triangles: $\underline{Y} \rightarrow \underline{Z} \rightarrow \underline{X} \rightarrow \underline{Y}[1]$ and $\underline{X} \rightarrow \underline{Z}^{\prime} \rightarrow \underline{Y} \rightarrow \underline{X} \overline{[1]}$.

Following [14, we say $\underline{X} \in \underline{\mathcal{C}} \widetilde{\widetilde{Q}}$ to be reachable if it belongs to a cluster-tilting subcategory which can be obtained by a sequence of mutations from $\underline{\mathcal{T}_{0}}$ with the mutations do not take at the objects in $\mathcal{T}^{\prime \prime}$. It is clear that any reachable object belongs to $\mathcal{U}$.

Following Theorem 4.1 of [14], we have the following result:

Theorem 3.6. Keep the notations as above. Then the cluster character $C C^{\prime}()$ gives a surjection from the set of equivalence classes of indecomposable reachable objects under equivalent modulo $\Gamma$ of $\underline{\mathcal{C}^{\widetilde{Q}}}$ to the set of clusters variables of the cluster algebra $\mathcal{A}(\Sigma)$.

Proof. By Theorem 3.5 the proof is similar as that of Theorem 4.1 in [14].

\section{Sign-COHEREnCE of g-VeCtors}

Keep the notations in Section 3. In this section, we will prove the sign-coherence of g-vectors for acyclic sign-skew-symmetric cluster algebras. For convenience, suppose that $\mathcal{A}(\Sigma)$ is an acyclic sign-skew-symmetric cluster algebra with principal coefficients at $\Sigma$.

Since $B$ is acyclic, $\left(\begin{array}{c}B \\ I_{n}\end{array}\right)$ is acyclic, too. We can construct an unfolding $(\widetilde{Q}, F, \Gamma)$ according to Theorem 2.5. It is easy to check that the corresponding seed $\widetilde{\Sigma}$ of $(\widetilde{Q}, F, \Gamma)$ is with principal coefficients, where $\widetilde{\Sigma}$ is the seed associate to $(Q, F)$. 
Proposition 4.1. Assume that $\underline{X}$ is an object of $\mathcal{U}$ given in Section 3.

(i) $C C(\underline{X})$ admits a $\mathbf{g}$-vector $\left(g_{i}\right)_{i \in \widetilde{Q}_{0} \backslash F}$ which is given by $g_{i}=\left[\right.$ ind $\left._{\mathcal{T}_{0}}(\underline{X}): \underline{T_{i}}\right]$ for each $i$.

(ii) $C C^{\prime}(\underline{X})$ admits a $\mathbf{g}$-vector $\left(g_{[i]}\right)_{[i] \in \overline{Q_{0}} \backslash \bar{F}}$ which is given by $g_{[i]}=\sum_{i^{\prime} \in[i]}\left[i n d_{\mathcal{T}_{0}}(\underline{X}): \underline{T_{i^{\prime}}}\right]$ for each $i$.

Proof. (i) Since the quiver $\widetilde{Q}$ is strongly almost finite, the proof is the same one as that of ([15], Proposition 3.6) using of the local finiteness of $\widetilde{Q}$.

(ii) is obtained immediately from (i).

Let $h \in \Gamma$ be either of finite order or without fixed points. Define $\underline{\mathcal{C}}^{\widetilde{Q}} h$ the $K$-linear category whose objects are the same as that of $\underline{\mathcal{C}} \underline{\widetilde{Q}}$, and whose morphisms consist of $\operatorname{Hom}_{\underline{\mathcal{C}}_{h}}(\underline{X}, \underline{Y})=$ $\bigoplus_{h^{\prime} \in \Gamma^{\prime}} H_{o m} m_{\mathcal{C}^{\tilde{Q}}}\left(h^{\prime} \cdot \underline{X}, \underline{Y}\right)$ for all objects $\underline{X}, \underline{Y}$. We view this category as a dual construction of the category $\mathcal{C}_{h}^{Q}$ in 12 .

Denote by $\underline{\mathcal{C}} \underline{\widetilde{Q}} h\left(\underline{\mathcal{T}_{0}}\right)$ the subcategory of $\underline{\mathcal{C}}_{h}^{\widetilde{Q}}$ consisting of all objects $\underline{T} \in \underline{\mathcal{T}_{0}}$.

Lemma 4.2. The category $\underline{\mathcal{C}}^{\widetilde{Q}}$ is Hom-finite if either (i) the order of $h$ is finite, or (ii) $Q$ has no fixed points under the action of $h \in \Gamma$.

Proof. The proof is similar to that of Lemma 6.1 in [12.

In the sequel, assume that $\underline{\mathcal{C}^{\widetilde{Q}}} h\left(\underline{\mathcal{T}_{0}}\right)$ is Hom-finite. Let $F: \underline{\mathcal{C}^{\widetilde{Q}}} \rightarrow \bmod \underline{\mathcal{C}^{\widetilde{Q}}} h\left(\underline{\mathcal{T}_{0}}\right)^{o p}$ be the functor mapping an object $X$ to the restricting of $\underline{\mathcal{C}}^{\widetilde{Q}} h(-, X)$ to $\underline{\mathcal{C}}^{\widetilde{Q}} h\left(\underline{\mathcal{T}_{0}}\right)$. For each indecomposable object $\underline{T}$ of $\underline{\mathcal{T}_{0}}$, denote by $S_{\underline{T}}$ the simple quotient of $F(\underline{T})$ via $S_{\underline{T}}\left(\underline{T}^{\prime}\right)=\operatorname{End}(\underline{T}) / J$ if $\underline{T}^{\prime} \cong \underline{T}$ and $S_{\underline{T}}\left(\underline{T}^{\prime}\right)=0$ if $\underline{T}^{\prime} \neq \underline{T}$, for any object $\underline{T}^{\prime}$ of $\underline{\mathcal{C}} \underline{\widetilde{Q}} h$, where $J$ is the Jacobson radical of $\operatorname{End}_{\underline{\mathcal{C}}_{h}}(\underline{T})$.

Lemma 4.3. Keep the notations as above. For any morphism $\tilde{f}: F(\underline{M}) \rightarrow F(\underline{N})$ in $\bmod \underline{\mathcal{C}} \underline{\widetilde{Q}} h\left(\underline{\mathcal{T}_{0}}\right)^{o p}$ with $M, N \in \mathcal{C}^{\widetilde{Q}}$, there exists $f: \underline{M} \rightarrow \underline{N}$ in $\underline{\mathcal{C}} \widetilde{Q} h$ such that $F(f)=\widetilde{f}$.

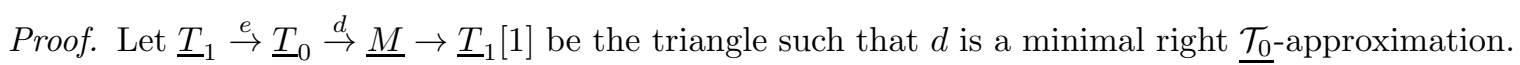
Since $\underline{\mathcal{T}_{0}}$ is a cluster tilting subcategory of $\underline{\mathcal{C}} \underline{\widetilde{Q}}$, we have $\underline{T}_{1} \in \underline{\mathcal{T}_{0}}$. Applying $F$ to the above triangle, we have $F\left(\underline{T}_{1}\right) \rightarrow F\left(\underline{T}_{0}\right) \rightarrow F(\underline{M}) \rightarrow 0$. Similarly, there is a triangle $\underline{T}_{1}^{\prime} \stackrel{e^{\prime}}{\rightarrow} \underline{T}_{0}^{\prime} \stackrel{d^{\prime}}{\rightarrow}$ $\underline{N} \rightarrow \underline{T}_{1}^{\prime}[1]$, and $F\left(\underline{T}_{1}^{\prime}\right) \rightarrow F\left(\underline{T}_{0}^{\prime}\right) \rightarrow F(\underline{N}) \rightarrow 0$. Since $\underline{T}_{0}, \underline{T}_{0}^{\prime}, \underline{T}_{1}, \underline{T}_{1}^{\prime} \in \underline{\mathcal{T}_{0}}$, it follows that $F\left(\underline{T}_{0}\right), F\left(\underline{T}_{0}^{\prime}\right), F\left(\underline{T}_{1}\right), F\left(\underline{T}_{1}^{\prime}\right)$ are projective in $\bmod \underline{\mathcal{C}}^{\widetilde{Q}}\left(\underline{\mathcal{T}}_{0}\right)^{o p}$. Thus, $\widetilde{f}$ can be lift to the following commutative diagram:

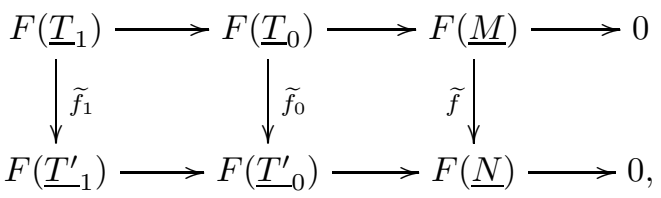

By the Yoneda Lemma, there exist $f_{1}: \underline{T}_{1} \rightarrow \underline{T}_{1}^{\prime}$ and $f_{0}: \underline{T}_{0} \rightarrow \underline{T}_{0}^{\prime}$ in $\underline{\mathcal{C}^{\widetilde{Q}}} h$ such that $F\left(f_{1}\right)=\widetilde{f}_{1}$ and $F\left(f_{0}\right)=\widetilde{f}_{0}$. Thus, $f_{1} \in \bigoplus_{h^{\prime} \in \Gamma^{\prime}} H o m_{\underline{\mathcal{C} \tilde{Q}}}\left(h^{\prime} \cdot \underline{T}_{1}, \underline{T}_{1}^{\prime}\right)$ and $f_{0} \in \bigoplus_{h^{\prime} \in \Gamma^{\prime}} H o m_{\underline{\mathcal{C} \tilde{Q}}}\left(h^{\prime} \cdot \underline{T}_{0}, \underline{T}_{0}^{\prime}\right)$ such that

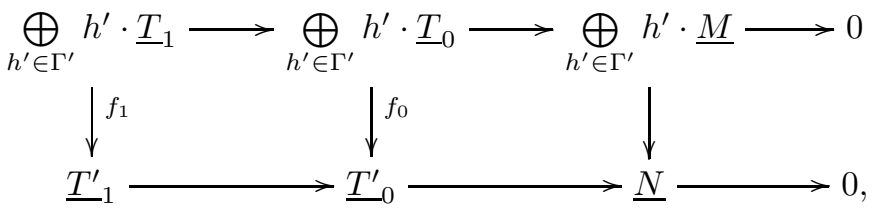


commutes. Since $\underline{\mathcal{C}^{\widetilde{Q}}} h\left(\underline{\mathcal{T}_{0}}\right)$ is Hom-finite, we may assume that only finite $h^{\prime} \in \Gamma^{\prime}$ appear in the upper triangle, which means that there exists a finite subset $I$ of $\Gamma^{\prime}$ such that

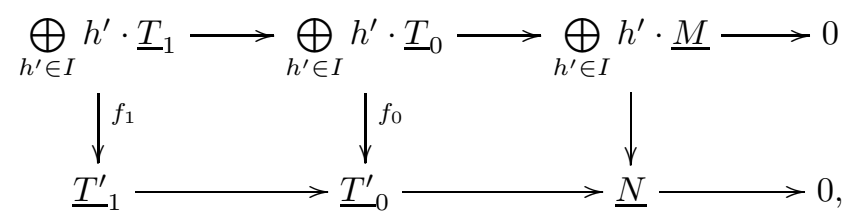

commutes. Thus, there exists $f \in \bigoplus_{h^{\prime} \in I} \operatorname{Hom}_{\underline{\mathcal{C}} \widetilde{Q}}\left(h^{\prime} \cdot \underline{T}_{0}, \underline{T}_{0}^{\prime}\right) \subseteq \bigoplus_{h^{\prime} \in \Gamma^{\prime}} H o m_{\underline{\mathcal{C}}_{\widetilde{Q}}}\left(h^{\prime} \cdot \underline{T}_{0}, \underline{T}_{0}^{\prime}\right)$ such that the above diagram commutes. This commutative diagram induces

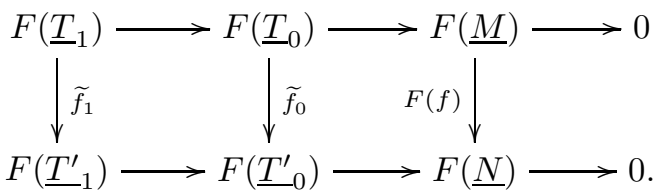

Therefore, we have $F(f)=\tilde{f}$.

Lemma 4.4. Assume that for $\underline{X} \in \mathcal{U}$, the category add $(\{h \cdot \underline{X} \mid h \in \Gamma\})$ is rigid. Let $\underline{T}_{1} \stackrel{f^{\prime}}{\rightarrow} \underline{T}_{0} \stackrel{f}{\rightarrow}$ $\underline{X} \rightarrow \underline{T}_{1}[1]$ be a triangle in $\underline{\mathcal{C}^{\widetilde{Q}}}$ with $f$ a minimal right $\underline{\mathcal{T}_{0}}$-approximation. If $\underline{X}$ has not any direct summand in $\underline{\mathcal{T}_{0}}[1]$, then

$$
F\left(\underline{T}_{1}\right) \stackrel{F\left(f^{\prime}\right)}{\longrightarrow} F\left(\underline{T}_{0}\right) \stackrel{F(f)}{\longrightarrow} F(\underline{X}) \rightarrow 0
$$

is a minimal projective resolution of $F(\underline{X})$.

Proof. Since $\underline{X}$ does not have direct summand in $\underline{\mathcal{T}_{0}}[1], f^{\prime}$ is right minimal. Otherwise, if $f^{\prime}$ is not right minimal, then $f^{\prime}$ has a direct summand as $\underline{T^{\prime}} \rightarrow 0$, thus $\underline{T^{\prime}}[1]$ is a direct summand of $\underline{X}$. This is a contradiction.

First, we prove that $F(f)$ is a projective cover of $F(\underline{X})$. For any projective representation $F(\underline{T}) e$ of $\underline{\mathcal{C}}^{\widetilde{Q}} h$ and surjective morphism $u: F(\underline{T}) e \rightarrow F(\underline{X}) \rightarrow 0$, where $\underline{T} \in \underline{\mathcal{C}}^{\widetilde{Q}} h, e \in \operatorname{End}_{\underline{\mathcal{C}}^{\widetilde{Q}}}(T)$ is an idempotent. By Yoneda Lemma, there exists $g \in \operatorname{Hom}_{\mathcal{C} \widetilde{Q}}(\underline{T}, \underline{X})$ such that $F(g)=u$. Since $F(\underline{T}) e$ and $F\left(\underline{T}_{0}\right)$ are projective, there exist $v: F\left(\underline{T}_{0}\right) \rightarrow F(\underline{T}) e$ and $w: F(\underline{T}) \rightarrow F\left(\underline{T}_{0}\right)$ such that $F(f)=F(g) v$ and $F(g)=F(f) w$. Thus, $F(f)=F(f) w v$. Similarly, by Yoneda Lemma, there exist $g^{\prime} \in \operatorname{Hom}_{\mathcal{C} \widetilde{Q}}\left(\underline{T}_{0}, \underline{T}\right)$ and $g^{\prime \prime} \in \operatorname{Hom}_{\mathcal{C} \widetilde{Q}}\left(\underline{T}, \underline{T}_{0}\right)$ such that $F\left(g^{\prime}\right)=v, F\left(g^{\prime \prime}\right)=w$. Thus, $F(f)=F(f) w v$ is equivalent to $f=f \circ g^{\prime \prime} \circ \bar{g}^{\prime}$. We may assume $g^{\prime \prime} \circ g^{\prime}=\left(g_{h^{\prime}}\right)_{h^{\prime} \in \Gamma^{\prime}}$, then $f=$

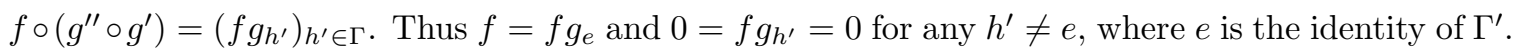
Further, since $f$ is a right minimal $\underline{\mathcal{T}_{0}}$-approximation and $h^{\prime} \underline{T}_{0} \in \underline{\mathcal{T}_{0}}$. Therefore, for any $e \neq h^{\prime} \in \Gamma$, $g_{h^{\prime}} \in J\left(h^{\prime} \underline{T}_{0}, \underline{T}_{0}\right)$ and $g_{e}$ is an isomorphism. Using Lemma 5.10 of [12], $g^{\prime \prime} \circ g^{\prime}=\left(g_{h^{\prime}}\right)_{h^{\prime} \in \Gamma^{\prime}}$ is an isomorphism. Thus, $F\left(\underline{T}_{0}\right)$ is a direct summand of $F(\underline{T}) e$.

Similarly, because $f^{\prime}$ is right minimal, $F\left(f^{\prime}\right)$ induces a projective cover of $k e r(F(f))$. Our result follows.

Inspired by (Proposition 2.1, 4]), (Lemma 3.58, [5]) and (Lemma 3.5, [15]), we have:

Lemma 4.5. Assume $\underline{X} \in \mathcal{U}$ such that add $(\{h \cdot \underline{X} \mid h \in \Gamma\})$ is rigid, and $\underline{T}_{1} \stackrel{f}{\rightarrow}_{\rightarrow} \underline{T}_{0} \stackrel{f}{\rightarrow} \underline{X} \rightarrow \underline{T}_{1}[1]$ is a triangle in $\underline{\mathcal{C}^{\widetilde{Q}}}$ and $f$ is a minimal right $\underline{\mathcal{T}}$-approximation. If $\underline{T}$ is a direct summand of $\underline{T}_{0}$ for indecomposable object $\underline{T} \in \underline{\mathcal{C}^{\widetilde{Q}}}$, then $h \cdot \underline{T}$ is not a direct summand of $\underline{T}_{1}$ for any $h \in \Gamma$.

Proof. By Lemma 2.3 and Lemma 2.4 of [12], we may assume that $h$ has no fixed points or finite order. By Lemma 4.2, $\underline{\mathcal{C}}^{\widetilde{Q}}$ is Hom-finite. For any $h^{\prime} \in \Gamma^{\prime}$ and $\underline{T}^{\prime} \in \underline{\mathcal{T}}$, applying $H_{o m} \underline{\mathcal{C}}\left(h^{\prime} \cdot \underline{T}^{\prime},-\right)$ 
to the triangle, we get

$$
H o m_{\underline{\mathcal{C} \widetilde{Q}}}\left(h^{\prime} \cdot \underline{T}^{\prime}, \underline{T}_{1}\right) \rightarrow H o m_{\underline{\mathcal{C} \widetilde{Q}}}\left(h^{\prime} \cdot \underline{T}^{\prime}, \underline{T}_{0}\right) \stackrel{H o m}{\underline{\mathcal{C} \widetilde{Q}}} \stackrel{\left(h^{\prime} \cdot \underline{T}^{\prime}, f\right)}{\longrightarrow} \operatorname{Hom}_{\underline{\underline{\mathcal{C}} \widetilde{Q}}}\left(h^{\prime} \cdot \underline{T}^{\prime}, \underline{X}\right) \rightarrow 0 .
$$

Since $f$ is minimal, we get a minimal projective resolution of $F(\underline{X})$,

$$
F\left(\underline{T}_{1}\right) \rightarrow F\left(\underline{T}_{0}\right) \rightarrow F(\underline{X}) \rightarrow 0 .
$$

To prove that $h \cdot \underline{T}$ is not a direct summand of $\underline{T}_{1}$, it suffices to prove that $F(\underline{T})$ is not a direct summand of $F\left(\underline{T}_{1}\right)$, or equivalently $\operatorname{Ext}^{1}\left(F(\underline{X}), S_{\underline{T}}\right)=0$, where $S_{\underline{T}}$ is the simple quotient of $F(\underline{T})$.

As $F\left(\underline{T}_{0}\right) \rightarrow F(\underline{X})$ is the projective cover of $F(\underline{X})$ and $F(\underline{T})$ is a direct summand of $F\left(\underline{T}_{0}\right)$, then there is a non-zero morphism $\tilde{p}: F(\underline{X}) \rightarrow S_{\underline{T}}$. For any $\widetilde{g}: F\left(\underline{T}_{1}\right) \rightarrow S_{\underline{T}}$, since $F\left(\underline{T}_{1}\right)$ is projective, there exists $\widetilde{q}: F\left(\underline{T}_{1}\right) \rightarrow F(\underline{X})$ such that $\widetilde{g}=\widetilde{p} \widetilde{q}$.

Let $T$ be a lifting of $\underline{T}$, by Lemma 3.1 , we have $T \in \mathcal{T}$ and $T$ is non-projective. Moreover, since $\underline{T}$ is indecomposable, we can choose $T$ is indecomposable. By Lemma 5.3 of [12, there is an admissible short exact sequence $0 \rightarrow Y \rightarrow T^{\prime} \rightarrow T \rightarrow 0$. Since $\mathcal{T}$ has no $\Gamma$-loop, by the dual version of Lemma 5.11 (2) of [12], and Lemma 3.1] we have $S_{\underline{T}} \cong F(\underline{Y}[1])$.

Thus, according to Lemma 4.3, lifting $\widetilde{q}, \widetilde{g}$ and $\widetilde{p}$ as $q, g, p$ in $\underline{\mathcal{C}} \underline{\widetilde{Q}} h$, where $q \in \bigoplus_{h^{\prime} \in \Gamma^{\prime}} H o m_{\mathcal{C}^{Q}}\left(h^{\prime}\right.$. $\left.\underline{T}_{1}, \underline{X}\right), g \in \bigoplus_{h^{\prime} \in \Gamma^{\prime}} H^{\prime} m_{\underline{\mathcal{C}^{\widetilde{Q}}}}\left(h^{\prime} \cdot \underline{T}_{1}, \underline{Y}[1]\right)$ and $p \in \bigoplus_{h^{\prime} \in \Gamma^{\prime}} H o m_{\underline{\mathcal{C}^{\widetilde{Q}}}}\left(h^{\prime} \cdot \underline{X}, \underline{Y}[1]\right)$. Since $\widetilde{g}=\tilde{p} \widetilde{q}$ and $\operatorname{Hom}(F(\underline{T}), F(\underline{Y}[1])) \cong \operatorname{Hom}_{\underline{\mathcal{C}}^{\widetilde{Q}}}(\underline{T}, \underline{Y}[1])$, we obtain $g=p \circ q$.

Since $\underline{\mathcal{C}^{\widetilde{Q}}} h$ is $H o m$-finite, we may assume that $g \in \bigoplus_{h^{\prime} \in I} H o m_{\underline{\mathcal{C}^{\widetilde{Q}}}}\left(h^{\prime} \cdot \underline{T}_{1}, \underline{Y}[1]\right)$ and $q \in \bigoplus_{h^{\prime} \in I} H o m_{\underline{\mathcal{C}^{\widetilde{Q}}}}\left(h^{\prime}\right.$. $\left.\underline{T}_{1}, \underline{X}\right)$ for a finite subset $I \subseteq \Gamma^{\prime}$. According to the composition of morphisms in $\underline{\mathcal{C}}^{\widetilde{Q}}, g=p \circ q$ means $g=p\left(\sum_{h^{\prime} \in I} h^{\prime} \cdot q\right)$, equivalently, we have the following commutative diagram:

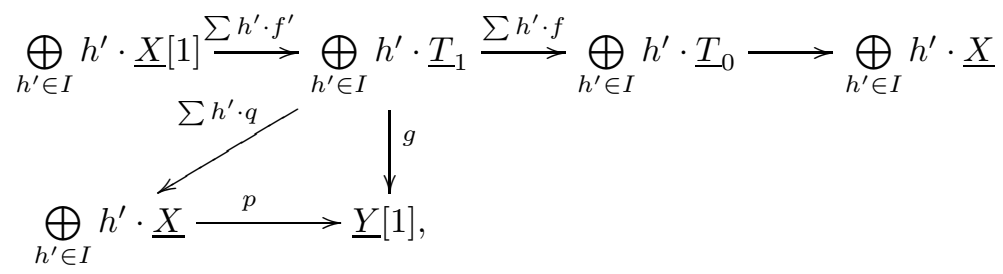

Since $\operatorname{add}(\{h \cdot X \mid h \in \Gamma\})$ is rigid, we have $g\left(\sum h^{\prime} \cdot f^{\prime}\right)=0$. Therefore, $g$ factor through $\sum h^{\prime} \cdot f$. Thus, in $\underline{\mathcal{C}^{\widetilde{Q}}} h, g$ factors through $f$. By the arbitrary of $g$, we get a surjective map $\operatorname{Hom}_{\underline{\mathcal{C}}_{\underline{Q}}}\left(\underline{T}_{0}, \underline{Y}[1]\right) \rightarrow \operatorname{Hom}_{\underline{\mathcal{C}}^{\widetilde{Q}} h}\left(\underline{T}_{1}, \underline{Y}[1]\right)$. Since $\operatorname{Hom}_{\underline{\mathcal{C}}_{h}}\left(\underline{T}_{i}, \underline{Y}[1]\right) \cong \operatorname{Hom}\left(F\left(\underline{T}_{i}\right), F(\underline{Y}[1])\right)=$ $\operatorname{Hom}\left(F\left(\underline{T}_{i}\right), S_{\underline{T}}\right)$ for $i=1,2$, we get $\operatorname{Hom}\left(F\left(\underline{T}_{0}\right), S_{\underline{T}}\right) \rightarrow \operatorname{Hom}\left(F\left(\underline{T}_{0}\right), S_{\underline{T}}\right)$ is surjective. Therefore, we obtain $\operatorname{Ext}^{1}\left(F(\underline{X}), S_{\underline{T}}\right)=0$. Our result follows.

Corollary 4.6. Let $\underline{X}$ be an object of $\underline{\mathcal{C}}$ such that add $(\{h \cdot \underline{X} \mid h \in \Gamma\})$ is rigid. If ind $\underline{\mathcal{T}_{0}}(\underline{X})$ has no negative coordinates, then $\underline{X} \in \underline{\mathcal{T}}$.

Proof. Assume $\underline{T}_{1} \stackrel{f^{\prime}}{\rightarrow} \underline{T}_{0} \stackrel{f}{\rightarrow} \underline{X} \rightarrow \underline{T}_{1}[1]$ is a triangle and $f$ is a minimal right $\mathcal{T}$-approximation. According to Lemma 4.5, $\underline{T}_{0}$ and $\underline{T}_{1}$ have no direct summands which have the same $\Gamma$-orbits. Further, since $i n d_{\underline{\mathcal{T}}}^{\prime}(\underline{X})$ has no negative components. Therefore, by the definition of $i n d_{\mathcal{T}_{0}}^{\prime}$, we obtain $\underline{T}_{1}=0$. Thus, $\underline{X} \cong \underline{T}_{0} \in \underline{\mathcal{I}}$.

Using the above preparation, we now can prove Conjecture 1.1 for all acyclic sign-skew-symmetric cluster algebras. The method of the proof follows from that of Theorem 3.7 (i) in [15].

Theorem 4.7. The conjecture 1.1 on sign-coherence holds for all acyclic sign-skew-symmetric cluster algebras. 
Proof. For any cluster $Z=\left\{z_{1}, \cdots, z_{n}\right\}$ of $\mathcal{A}(\Sigma)$, by Theorem 3.6, we associate a cluster tilting subcategory $\underline{\mathcal{T}}$ of $\underline{\mathcal{C}^{\widetilde{Q}}}$ which obtained by a series of mutations of $\underline{\mathcal{T}_{0}}$ and the mutations do no take at $\underline{\mathcal{T}^{\prime \prime}}$. Precisely, there are $n$ indecomposable objects $\left\{\underline{X_{j}} \mid j=1, \cdots, n\right\}$ such that $\underline{\mathcal{T}}=\operatorname{add}\left(\left\{h \cdot \underline{X_{j}} \mid j=\right.\right.$ $\left.1, \cdots, n\} \cup \underline{\mathcal{T}^{\prime \prime}}\right)$ and $C C^{\prime}\left(\underline{X_{i}}\right)=z_{i}$ for $i=1, \cdots, n$. By Proposition 4.1, the $g$-vector $g_{[1]}^{j}, \cdots, g_{[n]}^{j}$ of $z_{j}$ is given by $g_{[i]}^{j}=\sum_{i^{\prime} \in[i]}\left[i n d_{\mathcal{T}_{0}}\left(\underline{X_{j}}\right): \underline{T_{i^{\prime}}}\right]$.

Suppose that there exist $s$ and $s^{\prime}$ such that $g_{[i]}^{s}>0$ and $g_{[i]}^{s^{\prime}}<0$. Assume that $\underline{T}_{1}^{j} \rightarrow \underline{T^{j}}{ }_{0} \stackrel{f^{j}}{\rightarrow}$ $X_{j} \rightarrow \underline{T}^{j}{ }_{1}[1]$ be the triangle with $f^{j}$ is a minimal right $\underline{\mathcal{T}_{0}}$-approximation for $j=s, s^{\prime}$. Thus, there exist $h, h^{\prime} \in \Gamma$ such that $h \cdot \underline{T_{i}}$ (respectively, $h^{\prime} \cdot \underline{T_{i}}$ ) is a direct summand of $\underline{T}_{0}^{s}$ (respectively, $\underline{T}^{s^{\prime}}{ }_{1}$ ). Furthermore, in the triangle

$$
\bigoplus_{j=s, s^{\prime}} \underline{T}_{1}^{j} \rightarrow \bigoplus_{j=s, s^{\prime}} \underline{T}_{0}^{j} \stackrel{\bigoplus}{\stackrel{\oplus}{j=s, s^{\prime}} f^{j}} \bigoplus_{j=s, s^{\prime}} \underline{X}_{j} \rightarrow \bigoplus_{j=s, s^{\prime}} \underline{T}_{1}^{j}[1]
$$

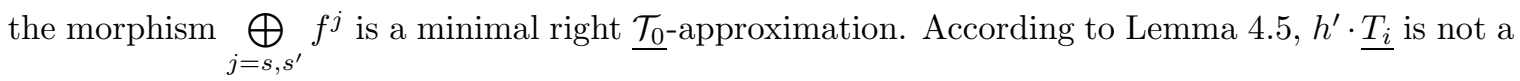
direct summand of $\underline{T^{s^{\prime}}}$ ince $h \cdot \underline{T_{i}}$ is a direct summand of $\underline{T}_{0}^{s}$. This is a contradiction. Our result follows.

\section{The RECURRENCE OF g-VECTORS}

Theorem 5.1. ([6] Conjecture 1.3 holds true for all finite rank skew-symmetric cluster algebras.

It is easy to see that the above theorem can be extended to the situation of infinite rank skewsymmetric cluster algebras, that is, we have:

Theorem 5.2. Conjecture 1.3 holds true for all infinite rank skew-symmetric cluster algebras.

We first give the following easy lemma.

Lemma 5.3. Let $(Q, F, \Gamma)$ be the unfolding of a matrix $B$ and $\mathcal{A}=\mathcal{A}(\Sigma(Q, F))$. For any sequence of orbits $\left(\left[i_{1}\right], \cdots,\left[i_{s}\right]\right)$ and $a \in Q_{0}$, there exist finite subsets $S_{j} \subseteq\left[i_{j}\right], j=1, \cdots, s$ such that $\prod_{k \in V_{s}} \mu_{k} \cdots \prod_{k \in V_{1}} \mu_{k}\left(x_{a}\right)=\widetilde{\mu}_{\left[i_{s}\right]} \cdots \widetilde{\mu}_{\left[i_{1}\right]}\left(x_{a}\right)$ for all finite subsets $V_{j}, j=1, \cdots, s$, satisfying $S_{j} \subseteq V_{j} \subseteq$ $\left[i_{j}\right], j=1, \cdots, s$.

Proof. Since $\widetilde{\mu}_{\left[i_{s}\right]} \cdots \widetilde{\mu}_{\left[i_{1}\right]}\left(x_{a}\right)$ is determined by finite vertices of $Q_{0}$, there exist finite subsets $S_{j} \subseteq$ $\left[i_{j}\right], j=1, \cdots, s$ such that $\prod_{k \in S_{s}} \mu_{k} \cdots \prod_{k \in S_{1}} \mu_{k}\left(x_{a}\right)=\widetilde{\mu}_{\left[i_{s}\right]} \cdots \widetilde{\mu}_{\left[i_{1}\right]}\left(x_{a}\right)$. Then for all finite subsets $V_{j}, j=1, \cdots, s$ satisfying $S_{j} \subseteq V_{j} \subseteq\left[i_{j}\right], j=1, \cdots, s$, we have $\prod_{k \in V_{s}} \mu_{k} \cdots \prod_{k \in V_{1}} \mu_{k}\left(x_{a}\right)=$ $\widetilde{\mu}_{\left[i_{s}\right]} \cdots \widetilde{\mu}_{\left[i_{1}\right]}\left(x_{a}\right)$.

Let $\mathcal{A}_{1}$ (respectively, $\mathcal{A}_{2}$ ) be the cluster algebra with principal coefficients at $\Sigma_{1}=(\tilde{X}, \tilde{Y}, Q)$ (respectively, $\left.\Sigma_{2}=\left(\widetilde{X}^{\prime}, \widetilde{Y}^{\prime}, \widetilde{\mu}_{[k]}(Q)\right)\right)$. For any sequence $\left(\left[i_{1}\right], \cdots,\left[i_{s}\right]\right)$ of orbits of $Q_{0}$ and $a \in Q_{0}=$ $\widetilde{\mu}_{[k]}\left(Q_{0}\right)$, denote $g^{Q, a}=\left(g_{i}^{Q}\right)_{i \in Q_{0}}$ (respectively, $\left.g^{\widetilde{\mu}_{[k]}(Q), a}=\left(g_{i}^{\widetilde{\mu}_{[k]}(Q)}\right)_{i \in Q_{0}}\right)$ be the g-vector of the cluster variable $\widetilde{\mu}_{\left[i_{s}\right]} \cdots \widetilde{\mu}_{\left[i_{1}\right]}\left(x_{a}\right)$ (respectively, $\widetilde{\mu}_{\left[i_{s}\right]} \cdots \widetilde{\mu}_{\left[i_{1}\right]} \widetilde{\mu}_{[k]}\left(x_{a}^{\prime}\right)$.

As a consequence of Theorem 5.2 we have the following property.

Proposition 5.4. Keep the notations as above. The following recurrence holds:

$$
g_{i}^{\widetilde{\mu}_{[k]}(Q)}= \begin{cases}-g_{i}^{Q} & \text { if } i \in[k] ; \\ g_{i}^{Q}+\sum_{k^{\prime} \in[k]}\left[b_{i k^{\prime}}\right]_{+} g_{k^{\prime}}^{Q}-\sum_{k^{\prime} \in[k]} b_{i k^{\prime}} \min \left(g_{k^{\prime}}^{Q}, 0\right) & \text { if } i \notin[k] .\end{cases}
$$


Proof. By Lemma 5.3, for $j=1, \cdots, s$, there exist finite subsets $S_{j}^{1} \subseteq\left[i_{j}\right]$ (respectively, $S_{j}^{2} \subseteq\left[i_{j}\right]$ ) such that $g^{Q, a}$ (respectively, $\left.g^{\widetilde{\mu}_{[k]}(Q), a}\right)$ is the $\mathbf{g}$-vector of the cluster variable $\prod_{t \in V_{s}^{1}} \mu_{t} \cdots \prod_{t \in V_{1}^{1}} \mu_{t}\left(x_{a}\right)$ (respectively, $\prod_{t \in V_{s}^{2}} \mu_{t} \cdots \prod_{t \in V_{1}^{1}} \mu_{t} \widetilde{\mu}_{[k]}\left(x_{a}^{\prime}\right)$ ) for all finite subsets $V_{j}^{1}$ (respectively, $V_{j}^{2}$ ) satisfying that $S_{j}^{1} \subseteq V_{j}^{1} \subseteq\left[i_{j}\right]$ (respectively, $S_{j}^{2} \subseteq V_{j}^{2} \subseteq\left[i_{j}\right]$ ). Choose $S_{j}=S_{j}^{1} \cup S_{j}^{2}$, we have $g^{Q, a}$ and $g^{\widetilde{\mu}_{[k]}(Q), a}$ as the g-vectors of the cluster variables $\prod_{t \in S_{s}} \mu_{t} \cdots \prod_{t \in S_{1}} \mu_{t}\left(x_{a}\right)$ and $\prod_{t \in S_{s}} \mu_{t} \cdots \prod_{t \in S_{1}} \mu_{t} \widetilde{\mu}_{[k]}\left(x_{a}^{\prime}\right)$ respectively.

For any finite subset $T \subseteq[k]$, denote by $\mathcal{A}^{T}$ the cluster algebra with principal coefficients at

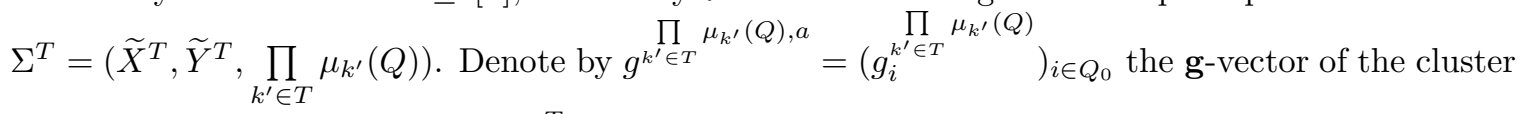
variable $\prod_{t \in S_{s}} \mu_{t} \cdots \prod_{t \in S_{1}} \mu_{t} \prod_{k^{\prime} \in T} \mu_{k^{\prime}}\left(x_{a}^{T}\right)$.

Since the cluster variable $\prod_{t \in S_{s}} \mu_{t} \cdots \prod_{t \in S_{1}} \mu_{t} \widetilde{\mu}_{[k]}\left(x_{a}^{\prime}\right)$ is only determined by finite vertices of $Q_{0}$, there exists a finite subset $S \subseteq[k]$ such that $g_{i}^{\widetilde{\mu}_{[k]}(Q)}=g_{i}^{\prod_{k^{\prime} \in T} \mu_{k^{\prime}}(Q)}$ for any finite set $T$ satisfying $S \subset T \subseteq[k]$.

Furthermore, by Theorem 5.2 for any subset $T \subseteq Q_{0}$, we have

$$
g_{i}^{\prod_{k^{\prime} \in T} \mu_{k^{\prime}}(Q)}= \begin{cases}-g_{i}^{Q} & \text { if } i \in T ; \\ g_{i}^{Q}+\sum_{k^{\prime} \in T}\left[b_{i k^{\prime}}\right]_{+} g_{k^{\prime}}^{Q}-\sum_{k^{\prime} \in T} b_{i k^{\prime}} \min \left(g_{k^{\prime}}^{Q}, 0\right) & \text { if } i \notin T .\end{cases}
$$

Therefore, the result holds.

Theorem 5.5. Conjecture 1.3 holds true for all acyclic sign-skew-symmetric cluster algebras. That is, let $B=\left(b_{[i][j]}\right) \in M_{a t_{n \times n}}(\mathbb{Z})$ be a sign-skew-symmetric matrix which is mutation equivalent to an acyclic matrix and let $t_{1}-[k]-t_{2} \in \mathbb{T}_{n}$ and $B^{2}=\mu_{[k]}\left(B^{1}\right)$. For $[a] \in\{[1], \cdots,[n]\}$ and $t \in \mathbb{T}_{n}$, assume $\mathbf{g}_{[a] ; t}^{B^{1} ; t_{1}}=\left(g_{[1]}^{t_{1}}, \cdots, g_{[n]}^{t_{1}}\right)$ and $\mathbf{g}_{[a] ; t}^{B^{2} ; t_{2}}=\left(g_{[1]}^{t_{2}}, \cdots, g_{[n]}^{t_{2}}\right)$, then

$$
g_{[i]}^{t_{2}}= \begin{cases}-g_{[k]}^{t_{1}} & \text { if }[i]=[k] \\ g_{[i]}^{t_{1}}+\left[b_{[i][k]}^{t_{1}}\right]_{+} g_{[k]}^{t_{1}}-b_{[i][k]}^{t_{1}} \min \left(g_{[k]}^{t_{1}}, 0\right) & \text { if }[i] \neq[k] .\end{cases}
$$

Proof. By Lemma 2.4 and Theorem 2.5, let $(Q, \Gamma)$ be an unfolding of $B$. Assume $t_{1}--t_{2}^{\prime} \cdots t_{s}^{\prime}--t$. By Theorem [2.9, we have $g_{[i]}^{t_{2}}=\sum_{i^{\prime} \in[i]} g_{i^{\prime}}^{\widetilde{\mu}_{[k]}(Q)}$ and $g_{[i]}^{t_{1}}=\sum_{i^{\prime} \in[i]} g_{i^{\prime}}^{Q}$. By Proposition 4.1 and Lemma 4.5. for $k^{\prime}, k^{\prime \prime} \in[k]$, both $g_{k^{\prime}}^{Q}$ and $g_{k^{\prime \prime}}^{Q}$ are non-negative or non-positive. Thus, $\sum_{k^{\prime} \in[k]} \min \left(g_{k^{\prime}}^{Q}, 0\right)=$ $\min \left(\sum_{k^{\prime} \in[k]} g_{k^{\prime}}^{Q}, 0\right)$. Using Proposition [5.4] if $[i]=[k]$, then

$$
g_{[i]}^{t_{2}}=\sum_{i^{\prime} \in[i]} g_{i^{\prime}}^{\widetilde{\mu}_{[k]}(Q)}=-\sum_{i^{\prime} \in[i]} g_{i^{\prime}}^{Q}=-g_{[i]}^{t_{1}} ;
$$

if $[i] \neq[k]$, since $\sum_{k^{\prime} \in[k]} \min \left(g_{k^{\prime}}^{Q}, 0\right)=\min \left(\sum_{k^{\prime} \in[k]} g_{K^{\prime}}^{Q}, 0\right)$ and $b_{[i][k]}^{t_{1}}=\sum_{i^{\prime} \in[i]} b_{i^{\prime} k}$, then

$$
g_{[i]}^{t_{2}}=\sum_{i^{\prime} \in[i]}\left(g_{i^{\prime}}^{Q}+\sum_{k^{\prime} \in[k]}\left[b_{i^{\prime} k^{\prime}}^{t_{1}}\right]_{+} g_{k^{\prime}}^{Q}-\sum_{k^{\prime} \in[k]} b_{i^{\prime} k^{\prime}}^{t_{1}} \min \left(g_{k^{\prime}}^{Q}, 0\right)\right)=g_{[i]}^{t_{1}}+\left[b_{[i][k]}^{t_{1}}\right]_{+} g_{[k]}^{t_{1}}-b_{[i][k]}^{t_{1}} \min \left(g_{[k]}^{t_{1}}, 0\right) .
$$

The result holds.

Acknowledgements: This project is supported by the National Natural Science Foundation of China (No.11671350 and No.11571173). 


\section{REFERENCES}

1. A. B. Buan, O. Iyama, I. Reiten and J. Scott, Cluster structures for 2-Calabi-Yau categories and unipotent groups. Compositio Math. 145 (2009), 1035-1079.

2. A. B. Buan, R. Marsh, M. Reineke, I. Reiten and G. Todorov, Tilting theory and cluster combinatorics, Adv. Math. 204 (2006), no.2, 572-618.

3. A. Berenstein, S. Fomin and A. Zelevinsky, Cluster algebras III: Upper bound and Bruhat cells. Duke Mathematical Journal. Vol.126, No.1(2005).

4. R. Dehy, B. Keller, On the combinatorics of rigid objects in 2-Calabi-Yau categories. Int. Math. Res. Not.(2008), doi:10.1093/imrn/rnn029.

5. L. Demonet, Categorification of skew-symmerizable cluster algebras. Algebr Represent Theory, 14, 1087-1162, 2011.

6. H. Derksen, J. Weyman, and A. Zelevinsky, Quivers with potentials and their representations II: applications to cluster algebras. J. Amer. Math. Soc., 23(3):749-790, 2010.

7. S. Fomin, A. Zelevinsky, Cluster algebras I: Foundations. J. Amer. Math. Soc. 15, 497-529(2002).

8. S. Fomin, A. Zelevinsky, Cluster algebras II: Finite type classification. Inven. Math. 154, 63-121(2003).

9. S. Fomin, A. Zelevinsky, Cluster algebras IV: Coefficients, Comp. Math. 143, 112-164, 2007.

10. C.J. Fu, B. Keller, On cluster algebras with coefficients and 2-CalabiCYau categories. Trans. Am. Math. Soc. 362(2), 859-895 (2010).

11. M. Gross, P. Hacking, S. Keel, M. Kontsevich, Canonical bases for cluster algebras, arXiv:1411.1394v1 [math.AG], 2014.

12. M. Huang, F. Li, Unfolding of sign-skew-symmetric cluster algebras and applications to positivity and Fpolynomials, arXiv:1609.05981

13. T. Nakanishi, A. Zelevinsky, On tropical dualities in cluster algebras, Contemp. Math. 565 (2012) 217-226.

14. P.-G. Plamondon, Cluster characters for cluster categories with infinite-dimensional morphism spaces, Adv. Math. 227 (2011), 1-39.

15. P.-G. Plamondon, Cluster algebras via cluster categories with infinite-dimensional morphism spaces, Compositio Math. 147, 1921-1954, 2011.

Peigen Cao

Department of Mathematics, Zhejiang University (Yuquan Campus), Hangzhou, Zhejiang 310027, P.R. China

E-mail address: peigencao@126.com

Min Huang

Department of Mathematics, Zhejiang University (Yuquan Campus), Hangzhou, Zhejiang 310027, P.R.China

E-mail address: minhuang1989@hotmail.com

FANG LI

Department of Mathematics, Zhejiang University (Yuquan Campus), Hangzhou, Zhejiang 310027, P.R.China

E-mail address: fangli@zju.edu.cn 